\title{
Linear increase of diploidy in human sperm with age: A four-colour FISH study
}

\author{
Mercè Bosch ${ }^{1}$, Osvaldo Rajmil ${ }^{2}$, Olga Martínez-Pasarell ${ }^{1}$, Josep Egozcue ${ }^{3}$ and \\ Cristina Templado*,1
}

${ }^{1}$ Departament de Biologia Cel.lular, Fisiologia i Immunologia, Facultat de Medicina, Universitat Autònoma de Barcelona, Bellaterra 08193, Spain; ${ }^{2}$ Servei d'Andrologia, Fundació Puigvert, Barcelona 08025, Spain;

${ }^{3}$ Departament de Biologia Cel.lular, Fisiologia i Immunologia, Facultat de Ciències, Universitat Autònoma de Barcelona, Bellaterra 08193, Spain

The aim of this study was to determine if donor age is associated with an increased incidence of diploidy and of disomy for the sex chromosomes and for chromosomes 6 and 21. We used simultaneous fluorescence in situ hybridisation (FISH) for chromosomes 6, 21, $\mathrm{X}$ and $\mathrm{Y}$ in sperm from 18 healthy donors, aged 24 - 74 years (mean 48.8years). A total of 194024 sperm were analysed, with a minimum of 10000 sperm scored for each donor. Our results indicate a significant increase of the level of diploidy $(P=0.002)$, and a marginal significance of total sex chromosome disomy $(P=0.055)$ with age. No increase was observed for disomies $X X, Y Y, X Y, 21$ or 6 . The percentages of increase for disomy and for diploidy ranged from 0.3 to $17 \%$ for each 10 -year period.

Chromosomes 6 and 21 did not segregate preferentially with the $X$ or $Y$ chromosomes. Our findings show a linear trend association between age and diploidy in human males. EuropeanJournal of Human Genetics (2001) 9, 533-538.

Keywords: aneuploidy; paternal age; sex ratio; spermatozoa; FISH

\section{Introduction}

Advanced maternal age is the most relevant aetiological factor involved in human chromosome abnormalities. The paternal contribution to aneuploid pregnancies that survive to term ranges from 10 to $100 \%$ depending on the chromosomes involved. ${ }^{1,2}$ Several epidemiological studies have reported a relationship between advanced paternal age and an increased risk of producing aneuploid offspring,,$^{3-7}$ but a paternal age effect has not been found by others. ${ }^{8,9}$ In these studies, maternally and paternally derived cases are seldom identified, and it becomes very difficult to assign a role to paternal age in the origin of aneuploidy.

Using molecular techniques, it is possible to determine whether the chromosome imbalance originated in the oocyte or the sperm. This allows the analysis of a possible relationship between advanced paternal age and trisomies of paternal origin. This approach has shown that only $10-30 \%$ of

\section{*Correspondence: Cristina Templado, Departament de Biologia,} Fisiologia i Immunologia, Facultat de Medicina, Universitat Autònoma de Barcelona, 08193 - Bellaterra, Barcelona, Spain. Tel: +34 93581 1905; Fax: +34 93581 1025; E-mail: cristina.templado@uab.es Received 1 February 2000; revised 23 March 2001; accepted 3 April 2001 autosomal trisomies (chromosomes 13, 14, 15, 21 and 22) originate during paternal meiosis. ${ }^{10,11}$ The paternal contribution to sex chromosome aneuploidy is more substantial; $100 \%$ of XYYs and half of XXYs are of paternal origin, ${ }^{5}$ and in $80 \%$ of Turner syndrome cases the missing $X$ is of paternal origin. ${ }^{12,13}$ Other studies have described significant increases in paternal age in uniparental disomy $15^{14}$ and in trisomy $18^{15}$ of paternal origin. However, MacDonald et al. (1994), ${ }^{16}$ Zaragoza et al. ${ }^{17}$ and Savage et al. ${ }^{18}$ observed no effect of increases of paternal age in their analyses of paternallyderived XXYs, XXXs, and acrocentric trisomies.

In spite of epidemiological and molecular studies, the influence of paternal age on human nondisjunction remains unclear. Both types of studies have limitations to isolate paternally-derived cases compared to maternally-derived cases when all trisomies are considered, due to their low frequency.

An alternative approach to overcome the problems encountered in evaluating a paternal age effect is the direct analysis of sperm chromosomes in aged men. Two- or three-colour fluorescence in situ hybridisation (FISH) assays have been used to determine if the frequency of aneuploid sperm increases with donor age. ${ }^{19-25}$ The sex chromosomes and chromosomes 
$1,6,8,12,13,14,17,18$ and 21 have been investigated for an age effect. All studies have detected an age effect for aneuploidy of the sex chromosomes, except one of them carried out in three men over 80 years of age. ${ }^{25}$ In the present study we used four-colour FISH with probes for chromosomes 6, 21, X and Y to analyse approximately 200000 sperm from 18 healthy sperm donors aged $24-74$ years (six of them over 60) to determine if donor age is associated with the incidence of disomy for chromosomes 6 and 21, and for the sex chromosomes, and with the incidence of diploidy.

\section{Materials and methods Sperm donors}

Semen samples were obtained from 18 healthy sperm donors aged 24-74 years free of exposures to any known mutagens, clastogens, radiation or drugs. Thirteen were of proven fertility (CM2, CM6, C7, C8, CM8, CM9, CM10, CM11, CM12, CM13, CM14, CM15, CM16) and five were of unknown fertility. Only four were smokers (CM1, CM4, CM6, CM10). All donors gave informed consent prior to the study. This research was approved by our institutional ethics committee.

\section{Sperm preparation}

Semen samples were collected in sterile containers and allowed to liquefy at $37^{\circ} \mathrm{C}$. The samples were washed three times with $0.05 \mathrm{M} \mathrm{KCl}$, fixed three times with methanol: acetic acid $(3: 1)$, smeared onto clean slides, and preserved at $-20^{\circ} \mathrm{C}$ until in situ hybridisation.

\section{Probes}

We applied simultaneous four-colour FISH, with centromeric probes for chromosomes 6 (1:1 mix of Spectrum green, CEP 6, and Spectrum orange, CEP 6, Vysis, Inc. Downers Grove, IL, USA), and X (Spectrum green, CEP X, Vysis, Inc), a DNA satellite III probe for the $\mathrm{Y}$ chromosome (Spectrum aqua, $\mathrm{Y}$ sat III, Vysis, Inc) and a locus specific DNA probe for chromosome 21 (Spectrum orange, LSI 21, Vysis, Inc). Chromosome 6 provided an internal control to characterise diploid and disomic cells and nonhybridised cells.

\section{Hybridisation procedure}

Sperm nuclei were washed for $3 \mathrm{~min}$ with standard sodium citrate (SSC) solution, dehydrated in an ethanol series, treated by slide incubation in a solution of $5 \mathrm{~mm}$ dithiothreitol (DTT), and denatured in 70\% formamide. Slides were hybridised for $72 \mathrm{~h}$ at $37^{\circ} \mathrm{C}$. Post-hybridisation washes followed manufacturer's instructions. DAPI counterstain (Vysis, Inc) and antifade were applied to the slides prior to observation.

\section{Data collection}

Slides were analysed under an Olympus AX70 epifluorescence microscope (Olympus Optical Co, Hamburg, Ger- many) equipped with a fluorescein isothiocyanate (FITC)/ Texas Red/4,6-diamino-2-phenylindole (DAPI) triple-band pass filter, and a single-band pass filter for FITC, Texas Red, and DAPI. The images were analysed with a Cytovision system (Applied Imaging, Sunderland, UK).

\section{Scoring criteria}

Only slides with a hybridisation efficiency of at least $99 \%$ were scored. Only intact and non overlapped sperm nuclei, identified as decondensed sperm by the presence of a tail, were evaluated. Two signals of the same colour were scored as two copies of the chromosome when they were comparable in brightness and size and separated from each other by a distance longer than the diameter of each signal. Nullisomy was considered when one of the signals was missing, and the nucleus contained one signal for the other chromosomes. We considered diploid those spermatozoa with one tail and two signals for each of the four chromosomes studied. Eight signals were scored in diploid sperm $v s$ five signals in disomic sperm.

\section{Statistical analysis}

A generalised additive model ${ }^{26}$ assuming that the data followed a Poisson distribution was fitted to each chromosome abnormality to explore its relation to age. Heterogeneity among donors resulted in overdispersion. This was tested by comparing the residual deviance reject to its degrees of freedom. If overdispersion was significant, a negative binomial distribution was assumed to perform the statistical tests. Observed proportions and fitted values from a smoothing spline function with $3^{\circ}$ of freedom are plotted to represent the non-linear relationship. Analysis of deviance was done to assess heterogeneity and trend (linear and quadratic), with age as a quantitative variable. Percentage increase in abnormalities for each 10 -year period, and its $95 \%$ confidence intervals were calculated from the coefficient of the linear model. Statistical and graphical analyses were performed using S-Plus. Chi-square (with Yates correction) with $1^{\circ}$ of freedom was used to compare the sex ratio in sperm disomic for chromosomes 6 or 21.

\section{Results}

A four-colour FISH assay was developed to simultaneously determine the sex ratio, aneuploidy frequencies for chromosomes 6, 21, X and $\mathrm{Y}$ and the rate of diploidy. A total of 194024 sperm nuclei were analysed in 18 normal men (age range $24-74$ years) with a minimum of 10000 sperm scored for each donor.

Table 1 shows the individual disomy and diploidy frequencies. We found interindividual differences in $\mathrm{XY}$, total sex chromosome and 21 disomy rates as well as in diploidy. The linear regression test for trend used only considers the variability caused by age, underestimating the overdispersion not explained by age, and probably related to 
other factors. When a significant heterogeneity was found with this test, an additional model based on the negative binomial distribution was used to account for it, which results in a decrease of significance. In spite of the loss of significance, the tendency to increased chromosome abnormality frequencies with age was maintained.

Our results indicate a significant tendency to an increase in the overall level of diploidy $(P=0.002)$ and a marginally significant trend in total sex chromosome disomy $(P=0.055)$ in relation to age (Table 2 ). When the model based on a negative binomial distribution was assumed, an increase in $\mathrm{XX}, \mathrm{YY}, \mathrm{XY}$, total sex chromosome, 6 and 21 disomy and diploidy $(13 \%, 0.3 \%, 11.7 \%, 8.6 \%, 11.4 \%, 5.9 \%$ and $17 \%$, respectively) was observed for each 10-year interval. The linear trend relating age, diploidy and aneuploidy frequencies for all chromosomes analysed is represented in Figure 1.

For each donor the ratio of X- to Y-bearing sperm was $1: 1$ as expected. The sex ratio in disomic sperm was 0.92 for chromosome 21 and 1.35 for chromosome 6 . We found no evidence that chromosomes 6 and 21 segregated preferentially with the $\mathrm{X}$-chromosome or the Y-chromosome $(P=0.37$ and $P=0.62$, respectively).

\section{Discussion}

This work was designed to determine if numerical chromosome abnormalities in human sperm are influenced by donor age. In previous studies, ${ }^{19-25}$ chromosomes $1,8,12,13,14$, $17,18,21, \mathrm{X}$ and $\mathrm{Y}$ were analysed by two- or three-colour FISH. The age of the 90 donors studied ranged from 22 to 83 years, but only six of them, which were analysed in two separate studies ${ }^{24,25}$, were over 60 . One study included three donors with an average age of 82 years, ${ }^{25}$ but the hybridis- ation efficiency was low (approximately 80\%) and the sperm cells scored were less than 5000 per donor due to the kind of the cells studied (testicular sperm cells). The present study was based on an age distribution that should facilitate the detection of an age effect, and 6/18 donors were over 60 .

As shown in Table 2, we found a significant interindividual heterogeneity in the frequencies of XY and 21 disomy, total sex chromosome disomy and diploidy. Significant interdonor variations have been reported in most FISH studies on paternal age on some chromosome aneuploidy rates. ${ }^{20-24}$ This heterogeneity, which could be related to the genetic background of the donors and to environmental exposures, makes it more difficult to detect trends in the numerical abnormality frequencies related to age.

The frequencies of disomy and diploidy, for the chromosomes studied, showed a tendency to increase with each 10 year interval, ranging from $0.3 \%$ to $17 \%$.

\section{Age vs disomy}

We detected a marginal significant linear increase in the frequency of total sex disomy with age $(P=0.055)$. Four previous FISH studies ${ }^{19-22}$ suggested that sex chromosome disomy increases with age in human sperm, affecting $X X$ and YY disomies, ${ }^{21} \mathrm{XX}, \mathrm{YY}$, and $\mathrm{XY}^{19}$ and $\mathrm{YY}$ disomy. ${ }^{20,22}$ However, the present study is the first to report a linear trend between sex chromosome disomy and increasing age. Disomy rates in our donors are consistent with those observed in human sperm karyotypes in three large series $^{27-29}$ in which chromosome 21 and the sex chromosomes showed higher aneuploidy frequencies than expected. However, no correlation, ${ }^{29}$ or negative correlation ${ }^{30}$ between the incidence of aneuploidy and donor age had been observed in sperm complement studies.

Table 1 Individual frequencies for disomy and diploidy in sperm from 18 normal men

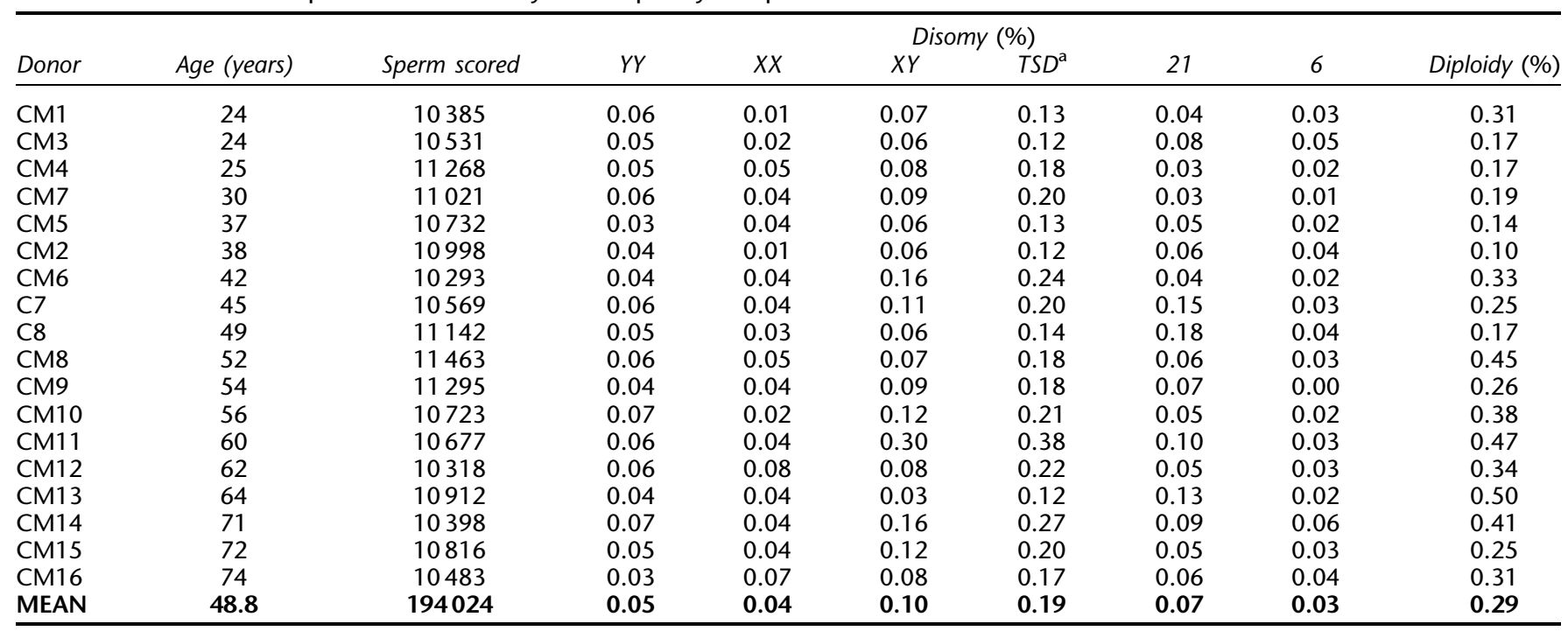

${ }^{\mathrm{a}} \mathrm{TSD}=$ total sex disomy. 
Table 2 Results of statistical analyses used to determine the association between male donor age and the rate of numerical chromosomal abnormalities (see text)

\begin{tabular}{|c|c|c|c|c|c|}
\hline \multirow{3}{*}{$\begin{array}{l}\text { Chromosome } \\
\text { abnormality }\end{array}$} & \multicolumn{4}{|c|}{ Linear trend in regression model } & \multirow{3}{*}{$\begin{array}{c}\text { Heterogeneity } \\
\text { P value }\end{array}$} \\
\hline & for 10 & $95 \%$ & & $P$ value & \\
\hline & & Less & Upper & & \\
\hline \multicolumn{6}{|l|}{ Disomy } \\
\hline$x X$ & 13.0 & -2.0 & 30.3 & 0.09 & 0.64 \\
\hline YY & 0.3 & -11.2 & 13.2 & 0.97 & 0.99 \\
\hline$X Y$ & 11.7 & -2.8 & 28.4 & 0.12 & $0.0001^{b}$ \\
\hline Total sex chr. & 8 & -0.2 & 18.1 & $0.055^{c}$ & $0.013^{\mathrm{b}}$ \\
\hline 21 & 11.4 & -3.2 & 28.1 & 0.13 & $0.007^{b}$ \\
\hline 6 & 5.9 & -10.3 & 25.0 & 0.50 & 0.51 \\
\hline Diploidy & 17.0 & 6.0 & 29.2 & $0.002^{d}$ & $<0.0001^{\mathrm{b}, \mathrm{d}}$ \\
\hline
\end{tabular}

${ }^{a}$ Confidence Interval; ${ }^{b}$ Negative binomial distribution was assumed; 'Marginal significance; ${ }^{d}$ Indicates significance of $P$ value for linear trend in spite of interdonor heterogeneity.

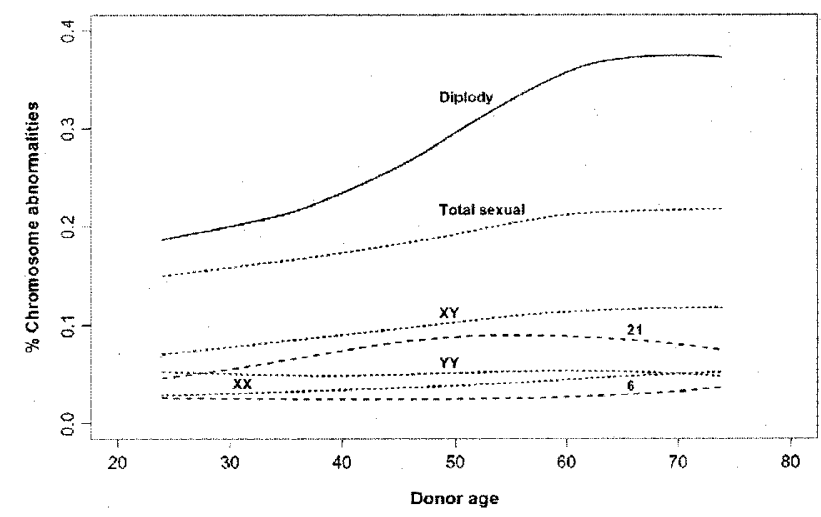

Figure 1 Relationship between age and frequencies of diploidy and of disomy for chromosomes $6,21, \mathrm{X}$ and $\mathrm{Y}$.

The sex bivalent may be particularly susceptible to pairing abnormalities since in normal male meiosis, a single chiasma is formed in the pseudoautosomal region. ${ }^{31-33}$ Some authors $^{5,34}$ demonstrated a strong correlation between failure of recombination and subsequent $\mathrm{XY}$ bivalent nondisjunction.

The age effect on disomy can be attributed to the precocious separation of the $\mathrm{X}$ and $\mathrm{Y}$ chromosomes or to an overall age-related increase in achiasmatic bivalents. Further research is warranted to determine which specific changes in paternal recombination are directly or indirectly associated with the age effect on nondisjunction.

As in two previous studies, ${ }^{23,24}$ no significant age effect was detected for 21 disomy frequency in our donors. Similarly, Yoon et al. ${ }^{35}$ and Savage et al. ${ }^{18}$ found no increased paternal age in their series of paternally derived 21 trisomy cases.

\section{Sex ratio in normal and disomic sperm}

We studied the frequencies of $\mathrm{X}$ - and Y-bearing sperm in haploid spermatozoa and in sperm disomic for chromosomes 6 and 21 in relation to age because an excess of males has been reported $^{6,18}$ among newborns with trisomy 21 . Griffin et al. ${ }^{36}$ found that chromosome 21 disomic sperm were more likely to be Y-bearing than X-bearing, and suggested a tendency of the extra chromosome 21 to preferentially segregate with the $\mathrm{Y}$ chromosome. Recently, Baumgartner et al. ${ }^{37}$ proposed that sperm with disomy 21 had an evident tendency to carry a Ychromosome, although their results were not significant $(P=0.07)$. We found no preferential segregation of the extra chromosome 21 with the X-chromosome or with the Ychromosome $(P=0.61)$ in our donors. This is in agreement with pooled data from human sperm complements with an extra chromosome $21 .^{29}$ This finding could indicate that a preferential selection of male conceptuses in which the extra chromosome 21 is paternally derived exists, and could explain the excess of males among trisomy 21 newborn babies.

\section{Age vs diploidy}

A significant association between donor age and diploidy was detected $(P<0.002)$ in our study, while some of the age studies performed so far had found no association between age and diploidy frequency. ${ }^{19,20,22,25}$ In other studies, this parameter was not even analysed ${ }^{23}$ or discussed. ${ }^{21,24}$ The use of simultaneous four-colour FISH allowed for a more accurate scoring of diploidy, although it is not possible to discard that these differences in the incidence of diploidy be caused by heterogeneity among donors. Moreover, our data are in agreement with the high percentage of triploids of paternal origin (among which over 10\% originate from diploid sperm). ${ }^{38}$ Significant frequencies of diploid sperm have also been found in some carriers of balanced chromosome reorganisations, ${ }^{39}$ in fathers of children with Down syndrome, ${ }^{40}$ and in infertile patients, specially oligoasthenoteratozoospermic patients. ${ }^{41,42}$

One possible explanation for this increase of diploid sperm frequency in older men would be the tendency of these individuals to show synaptic anomalies related to a progressively deteriorating testicular environment. ${ }^{43,44}$ This would result in an increase in segregation defects during meiosis I, leading to an arrest of cytokinesis, and to the subsequent formation of diploid secondary spermatocytes, spermatids and spermatozoa. ${ }^{44}$

\section{Conclusions}

Our results indicate that age is associated with an increased frequency of diploidy, and suggest an association with increased sex chromosome disomy. Diploidy shows the highest rate of increase for each 10-year interval (17\%), indicating that this abnormality is the most susceptible to increase with age.

The slight increases in sex chromosome disomies and diploidy detected by us may not suggest an immediate, 
significant clinical effect. However, increased frequencies of chromosome 21 disomy have been found in spermatozoa from fathers of Down patients of paternal origin, ${ }^{40,45}$ and an increase of XY disomy have been detected in fathers of Turner patients. ${ }^{46}$ In at least one case, a man with an elevated frequency of 24,XY spermatozoa fathered a pregnancy with a 47,XXY karyotype, detected by prenatal diagnosis. ${ }^{47}$ Moreover, taking into account that a large proportion of conceptuses are triploid, ${ }^{44,48}$ the slight increases in numerical chromosome abnormalities detected in spermatozoa could have more clinical importance than has been recognised.

\section{Acknowledgments}

This work received financial support from Ministerio de Educación y Cultura (DGESEIC, project PB98-0893) and Generalitat de Catalunya (CIRIT, 1999 SGR-00104), Spain. Mercè Bosch was the recipient of a grant from Ministerio de Educación y Cultura (FPI, AP96).

\section{References}

1 Hassold T, Hunt PA, Sherman S: Trisomy in humans: incidence, origin and etiology. Curr Opin Genet Dev 1993; 3: 398-403.

2 Abruzzo MA, Hassold TJ: Etiology of nondisjunction in humans. Environ Mol Mutagen 1995; 25: 38- 47.

3 Carothers AD, Collyer S, De Mey R et al: Parental age and birth order in the aetiology of some sex chromosome aneuploidies. Ann Hum Genet 1978; 41: 277-287.

4 Stene E, Stene J, Stengel-Rutkowski S: A reanalysis of the New York State prenatal diagnosis data on Down's syndrome and paternal age effects. Hum Genet 1987; 77: 299-302.

5 Lorda-Sanchez I, Binkert F, Maechler M et al: Reduced recombination and paternal age effect in Klinefelter syndrome. Hum Genet 1992; 89: 524-530.

6 Petersen MB, Antonarakis SE, Hassold TJ et al: Paternal nondisjunction in trisomy 21: excess of male patients. Hum Mol Genet 1993; 2: 1691-1695.

7 Thepot F, Mayaux MJ, Czyglick F et al: Incidence of birth defects after artificial insemination with frozen donor spermatozoa: a collaborative study of the French CECOS Federation on 11,535 pregnancies. Hum Reprod 1996; 11: 2319-2323.

8 Cross PK, Hook EB: An analysis of paternal age and 47,+21 in 35,000 new prenatal cytogenetic diagnosis data from the New York State Chromosome Registry: no significant effect. Hum Genet 1987; 77: $307-316$.

9 Hook EB, Cross PK: Extra structurally abnormal chromosomes (ESAC) detected at amniocentesis: frequency in approximately 75,000 prenatal cytogenetic diagnoses and associations with maternal and paternal age. Am J Hum Genet 1987; 40: 83-101.

10 Hassold T, Abruzzo M, Adkins K et al: Human aneuploidy: incidence, origin, and etiology. Environ Mol Mutagen 1996; 28 $167-175$.

11 Wyrobek AJ, Aardema M, Eichenlaub-Ritter U et al: Mechanisms and targets involved in maternal and paternal age effects on numerical aneuploidy. Environ Mol Mutagen 1996; 28: 254-264.

12 Lorda-Sanchez I, Binkert F, Maechler M et al: Molecular study of 45, X conceptuses: correlation with clinical findings. Am J Med Genet 1992; 42: 487-490.

13 Martínez-Pasarell O, Templado C, Egozcue J et al: PCR protocol to detect parental origin and hidden mosaicism in sex chromosome aneuploidies. Horm Res 1999; 51: 248-252.

14 Robinson WP, Lorda-Sanchez I, Malcolm S et al: Increased parental ages and uniparental disomy 15: a paternal age effect? Eur J Hum Genet 1993; 1: 280-286.
15 Ya-gang X, Robinson WP, Spiegel R et al: Parental origin of the supernumerary chromosome in trisomy 18 . Clin Genet 1993; 44: $57-61$.

16 MacDonald M, Hassold T, Harvey J et al: The origin of 47,XXY and 47,XXX aneuploidy: heterogeneous mechanisms and role of aberrant recombination. Hum Mol Genet 1994; 3: 1365-1371.

17 Zaragoza MV, Jacobs PA, James RS et al: Nondisjunction of human acrocentric chromosomes: studies of 432 trisomic fetuses and liveborns. Hum Genet 1994; 94: 411-417.

18 Savage AR, Petersen MB, Pettay D et al: Elucidating the mechanisms of paternal non-disjunction of chromosome 21 in humans. Hum Mol Genet 1998; 7: 1221-1227.

19 Griffin DK, Abruzzo MA, Millie EA et al: Nondisjunction in human sperm: evidence for an age effect of increasing paternal age. Hum Mol Genet 1995; 4: 2227-2232.

20 Martin RH, Spriggs E, Ko E et al: The relationship between paternal age, sex ratios, and aneuploidy frequencies in human sperm, as assessed by multicolor FISH. Am J Hum Genet 1995; 57: $1395-1399$.

21 Robbins WA, Baulch JE, Moore D et al: Three-probe fluorescence in situ hybridization to assess chromosome $\mathrm{X}, \mathrm{Y}$, and 8 aneuploidy in sperm of 14 men from two healthy groups: evidence for a paternal age effect on sperm aneuploidy. Reprod Fertil Dev 1995; 7: 799-809.

22 Kinakin B, Rademaker A, Martin R: Paternal age effect of YY aneuploidy in human sperm, as assessed by fluorescence in situ hybridization. Cytogenet Cell Genet 1997; 78: 116-119.

23 McInnes B, Rademaker AW, Martin RH: Donor age and the frequency of disomy for chromosomes 1, 13, 21 and structural abnormalities in human spermatozoa using multicolor fluorescence in-situ hybridization. Hum Reprod 1998; 13: 2489-2494.

24 Rousseaux S, Hazzouri M, Pelletier R et al: Disomy rates for chromosomes 14 and 21 studied by fluorescent in-situ hybridization in spermatozoa from three men over 60 years of age. Mol Hum Reprod 1998; 4: 695-699.

25 Guttenbach M, Köhn FM, Engel W, Schmid M: Meiotic nondisjunction of chromosomes $1,17,18, \mathrm{X}$ and $\mathrm{Y}$ in men more than 80 years of age. Biol Reprod 2000; 63: 1727-1729.

26 Hastie TJ, Tbshirani RJ (eds): Generalized additive models. Chapman Hall, London, 1990.

27 Martin RH, Rademaker A: The frequencies of aneuploidy among individual chromosomes in 6,821 human sperm complements. Cytogenet Cell Genet 1990; 53: $103-107$.

28 Brandriff $B$, Gordon L: Human sperm cytogenetics and the onecell zygote; in Allen JW, Bridges BA, Lyon MF, Moses MJ, Rusell LB (eds): Biology of Mammalian Germ Cell Mutagenesis. Cold Spring Harbor Laboratory Press, 1990, pp 183-194.

29 Templado C, Marquez C, Munne S et al: An analysis of human sperm chromosome aneuploidy. Cytogenet Cell Genet 1996; 74: 194-200.

30 Martin RH, Rademaker AW: The effect of age on the frequency of sperm chromosomal abnormalities in normal men. Am J Hum Genet 1987; 41: $484-492$.

31 Burgoyne PS: Genetic homology and crossing over in the $\mathrm{X}$ and Y chromosomes of mammals. Hum Genet 1982; 61: 85 -90.

32 Goodfellow PJ, Darling SM, Thomas NS et al: A pseudoautosomal gene in man. Science 1986; 7: $740-743$.

33 Page DC, Bieker K, Brown LG et al: Linkage, physical mapping, and DNA sequence analysis of pseudoautosomal loci on the human X and Y chromosomes. Genomics 1987; 1: 243-256.

34 Hassold T, Sherman SL, Pettay D et al: XY chromosome nondisjunction in man is associated with diminished recombination in the pseudoautosomal region. Am J Hum Genet 1991; 49: $253-260$.

35 Yoon PW, Freeman SB, Sherman SL et al: Advanced maternal age and the risk of Down syndrome characterized by the meiotic stage of chromosomal error: a population-based study. Am J Hum Genet 1996; 58: 628-633. 
36 Griffin DK, Abruzzo MA, Millie EA et al: Sex ratio in normal and disomic sperm: evidence that the extra chromosome 21 preferentially segregates with the Y chromosome. Am J Hum Genet 1996; 59: 1108-1113.

37 Baumgartner A, Van Hummelen P, Lowe XR et al: Numerical and structural chromosomal abnormalities detected in human sperm with a combination of multicolor FISH assays. Environ Mol Mutagen 1999; 33: 49-58.

38 Zaragoza MV, Surti U, Redline RW et al: Parental origin and phenotype of triploidy in spontaneous abortions: predominance of diandry and association with the partial hydatidiform mole. Am J Hum Genet 2000; 66: 1807 - 1820.

39 Van Hummelen P, Manchester D, Lowe X et al: Meiotic segregation, recombination, and gamete aneuploidy assessed in a $\mathrm{t}(1 ; 10)(\mathrm{p} 22.1 ; \mathrm{q} 22.3)$ reciprocal translocation carrier by three- and four-probe multicolor FISH in sperm. Am J Hum Genet 1997; 61: 651-659.

40 Blanco J, Gabau E, Gomez D et al: Chromosome 21 disomy in the spermatozoa of the fathers of children with trisomy 21 in a population with a high prevalence of Down's syndrome. Increased incidence in cases of paternal origin. Am J Hum Genet 1998; 63: $1067-1072$.

41 Aran B, Blanco J, Vidal F et al: Screening for abnormalities of chromosomes $\mathrm{X}, \mathrm{Y}$, and 18 and for diploidy in spermatozoa from infertile men participating in an in vitro fertilizationintracytoplasmic sperm injection program. Fertil Steril 1999; 72: $696-701$.
42 Pang MG, Hoegerman SF, Cuticchia AJ et al: Detection of aneuploidy for chromosomes $4,6,7,8,9,10,11,12,13,17,18$, $21, X$ and $Y$ by fluorescence in-situ hybridization in spermatozoa from nine patients with oligoasthenoteratozoospermia undergoing intracytoplasmic sperm injection. Hum. Reprod 1999; 14: $1266-1273$.

43 Mroz K, Hassold TJ, Hunt PA. Meiotic aneuploidy in the XXY mouse: evidence that a compromised testicular environment increases the incidence of meiotic errors. Hum Reprod 1999; 14: $1151-1156$.

44 Egozcue S, Blanco J, Vendrell JM et al: Human male infertility: chromosome anomalies, meiotic disorders, abnormal spermatozoa and recurrent abortion. Hum Reprod Update 2000; 6: 93 105.

45 Soares SR, Templado C, Blanco J et al: Numerical chromosome abnormalities in the spermatozoa of the fathers of children with trisomy 21 of paternal origin: generalised tendency to meiotic non-disjunction. Hum Genet 2001 108: 134-139.

46 Martínez-Pasarell O, Nogués C, Bosch M et al: Analysis of sex chromosome aneuploidy in sperm from fathers of Turner syndrome patients. Hum Genet 1999; 104: 345 - 349 .

47 Moosani N, Chernos J, Lowry RB et al: A 47,XXY fetus resulting from ICSI in a man with an elevated frequency of $24, \mathrm{XY}$ spermatozoa. Hum Reprod 1999; 14: 1137-1138.

48 Hassold T, Chen N, Funkhouser J et al: A cytogenetic study of 1000 spontaneous abortions. Ann Hum Genet 1980; 2: 151-178. 